\title{
Florística do estrato herbáceo-subarbustivo de um campo limpo úmido em Brasília, Brasil
}

\author{
Cássia Beatriz Rodrigues Munhoz ${ }^{1,3}$ \& Jeanine Maria Felfili ${ }^{2}$
}

Biota Neotropica v7 (n3) - http://www.biotaneotropica.org.br/v7n3/pt/abstract?article +bn03707032007

\author{
Recebido em 04/04/07 \\ Versão Reformulada recebida em 08/10/07 \\ Publicado em 24/10/07
}

${ }^{1}$ Universidade Católica de Brasília, QS 07 Lote 01 sala E-10, Taguatinga Sul, CEP 72030-170, Brasília, DF, Brasil

${ }^{2}$ Departamento de Engenharia Florestal, Universidade de Brasília, CP 04357, CEP 70919-970, Brasília, DF, Brasil http://www.unb.br

${ }^{3}$ Autor para correspondência: Cássia Beatriz Rodrigues Munhoz,

e-mail: cassia@ucb.br, http://www.ucb.br

\begin{abstract}
Munhoz, C.B.R \& Felfili, J.M. Floristic of the herbaceous and subshrub layer of a Moist Grassland in Brasília, Brazil. Biota Neotrop. Sep/Dez 2007 vol. 7, no. 3 http://www.biotaneotropica.org.br/v7n3/pt/ abstract?article+bn03707032007. ISSN 1676-0603.

Little is known on the floristic richness of the herb-subshrub layer of the moist grassland, a grassy field vegetation within the Cerrado biome, although herbs and subshrubs are the major components of the Cerrado flora. The objective of this work was to characterize the floristic richness of the herb-subshrub layer of a moist grassland, in 16 ha in Fazenda Água Limpa (FAL), Brasília, DF (15 $56^{\prime}$ to $15^{\circ} 59^{\prime} \mathrm{S}$ and $47^{\circ} 55^{\prime}$ to $47^{\circ} 58^{\prime} \mathrm{W}$ ) and to compare with it with other Savana-like vegetations in central and southeastern Brazil. Vouchers were collected at quarterly intervals, from September 1999 to February 2001 along transects established in order to cover most of the study-area. A total of 197 species in 105 genera and 41 families was recorded. A species of Syngonanthus (Eriocaulaceae), probably new to science was found. The richest families were Poaceae (39 species); Cyperaceae (24); Asteraceae (21); Polygalaceae (15); Eriocaulaceae (12); Xyridaceae and Melastomataceae (8); and Fabaceae (7). Similarities between this moist grassland and 12 other sites, measured with the Sorensen index, were low, varying from $3.3 \%$ to $37.4 \%$. The low similarities between the compared sites may be related to the differences in soil water saturation or differences in the size of the area covered by grasslands in each site. The ordination analysis readily distinguished dry from humid sites and suggested that geographic distance between the sites can also affect floristic heterogeneity in the herbaceous-subshrub component.

Keywords: cerrado, floristic composition, grassland, herbaceous layer, savanna, wetland.

\section{Resumo}

Munhoz, C.B.R \& Felfili, J.M. Florística do estrato herbáceo-subarbustivo de um campo limpo úmido em Brasília, Brasil. Biota Neotrop. Sep/Dez 2007 vol. 7, no. 3 http://www.biotaneotropica.org.br/v7n3/pt/ abstract?article+bn03707032007. ISSN 1676-0603.

A riqueza florística do estrato herbáceo-arbustivo de campo limpo úmido, uma vegetação campestre do bioma Cerrado, é pouco conhecida, embora as ervas e subarbustos sejam o maior componente na vegetação de Cerrado. O presente trabalho teve por objetivo caracterizar a riqueza florística da camada herbáceo-subarbustiva de um campo limpo úmido, com aproximadamente 16 ha, localizado na Fazenda Água Limpa (FAL), Brasília, DF (15 56' a $15^{\circ} 59^{\prime} \mathrm{S}$ e $47^{\circ} 55^{\prime}$ a $47^{\circ} 58^{\prime} \mathrm{W}$ ), além de comparar a sua flora herbáceo-arbustiva com a de outras áreas do bioma Cerrado no Brasil central e sudeste. Material botânico fértil foi coletado quinzenalmente, de setembro de 1999 a fevereiro de 2001, ao longo de trilhas distribuídas de modo a percorrer a maior extensão da área possível. Foram registradas 197 espécies, distribuídas em 105 gêneros e 41 famílias, tendo sido provavelmente descoberta uma nova espécie de Syngonanthus (Eriocaulaceae). As oito famílias com o maior número de espécie foram Poaceae (39 espécies); Cyperaceae (24); Asteraceae (21); Polygalaceae (15); Eriocaulaceae (12); Xyridaceae e Melastomataceae (8) e Fabaceae (7). A similaridade florística, medida com índice de Sørensen, entre o campo limpo úmido estudado e as outras 12 áreas de Cerrado foram baixas variando de 3,3\% a 37,4\%. As baixas similaridades entre as áreas comparadas podem estar relacionadas com as diferenças de saturação hídrica do solo ou diferenças no tamanho das áreas de Cerrado remanescentes. A ordenação separou claramente as áreas úmidas das áreas secas e sugere que a distância geográfica entre as mesmas também pode ter influenciado a heterogeneidade florística no componente herbáceo-subarbustivo.
\end{abstract}

Palavras-chave: cerrado, campo limpo úmido, composição florística, estrato herbáceo, áreas úmidas. 


\section{Introdução}

As fisionomias campestres de Cerrado caracterizam-se pelo predomínio do estrato herbáceo-subabustivo e de pequenos arbustos, e ocorrem normalmente na transição entre as matas e o cerrado sensu stricto, desempenhando uma importante ligação entre essas fisionomias. Os campos limpos ocorrem sobre solos com gradações de umidade, com faixas de campo limpo úmido onde o lençol freático é superficial, especialmente em áreas de nascentes, em encostas e nos fundos dos vales, seguido por campo limpo seco em solo bem drenado (Ribeiro \& Walter 1998).

A Fazenda Água Limpa, onde este estudo foi conduzido, encontrase na Área de Proteção Ambiental Gama e Cabeça de Veado, e está entre as áreas da Reserva da Biosfera do Cerrado no Distrito Federal, reconhecida pelo programa $\mathrm{MaB}$ - Homem e Biosfera da UNESCO. Nesta área de proteção ambiental os campos limpos úmidos ocorrem bordeando as matas de galeria, em solos hidromórficos, Gleis e orgânicos turfosos (Felfili et al. 2002) e constituem um ecossistema de grande relevância, sendo considerado como bacia coletora das águas absorvidas pelos platôs adjacentes. No Cerrado, a área estimada de campos úmidos estacionalmente inundáveis sobre solos hidromórficos é de 2,3\% e sobre solo do tipo Glei Húmico de 0,2\% (Reatto et al. 1998).

A camada herbáceo-arbustiva de Cerrado, com predominância de ervas graminóides, apresenta grande riqueza de espécies e ainda carece de estudos sobre a sua ecologia e composição florística (Tannus \& Assis 2004, Munhoz \& Felfili 2004, Munhoz \& Felfili 2006a). Para o bioma Cerrado, Mendonça et al. (1998) encontraram uma proporção de 4,5:1 de espécies arbustivo-herbáceas para arbóreas e para a Chapada Pratinha, onde se encontra o Distrito Federal, Felfili et al. (1994) encontraram uma razão de 3:1.

A flora herbáceo-graminosa das savanas é bem distinta da dos demais biomas e consiste de muitas espécies quase exclusivas desse ecossistema (Sarmiento 1992). Estudos florísticos e fitossociológicos que analisaram o componente herbáceo de Cerrado no nível local (Goodland 1969, Mantovani \& Martins 1993, Felfili et al. 1994, Silva \& Nogueira 1999, Munhoz \& Felfili 2006b), encontraram um predomínio de Poaceae na frequiência das espécies, principalmente dos gêneros Andropogon, Axonopus, Echinolaena, Paspalum e Trachypogon.

São escassos os estudos que compararam a similaridade específica do componente herbáceo-arbustivo entre áreas de Cerrado, quer seja entre fitofisionomias semelhantes ou distintas. Em uma comparação das floras de áreas de cerradão, cerrado sensu stricto e campo cerrado situados na Reserva Ecológica Pé-de-Gigante no estado de São Paulo, Batalha \& Mantovani (2001) encontraram maior similaridade entre o componente lenhoso das três fitofisionomias do que para o herbáceosubarbustivo. Araújo et al. (2002), comparando os levantamentos de quatro veredas em Uberlândia no estado de Minas Gerais, encontram maior sobreposição de espécies entre as porções mais drenadas das veredas do que entre as mais úmidas, e concluíram que as diferenças de umidade nestas áreas são determinantes na distribuição das espécies. Tannus \& Assis (2004) também observaram uma acentuada diferença florística entre um campo úmido e um campo sujo adjacente localizado no estado de São Paulo.

Variações regionais nos padrões de distribuição de espécies da flora vascular de Cerrado foram encontradas por Batalha \& Martins (2007), que compararam a flora do Parque Nacional de Emas (PNE), localizado a sudoeste do estado de Goiás, com as floras de outras três áreas disjuntas de Cerrado, situadas a sudoeste do estado de São Paulo. Estes autores também encontraram uma distribuição de frequiência de espécies por família significativamente diferente entre o componente herbáceo do PNE e o do Cerrado como um todo.
O presente trabalho teve por objetivo caracterizar a riqueza florística de um campo limpo úmido localizado na Fazenda Água Limpa no Distrito Federal, além de comparar sua flora herbáceo-arbustiva com a de outras áreas de Cerrado, contribuindo para estudos fitogeográficos e fornecendo subsídios para futuros trabalhos na área.

\section{Material e Métodos}

\section{Localização e descrição geral da área}

Este estudo foi conduzido em uma área de campo limpo úmido, na Fazenda Água Limpa - FAL (15 $56^{\prime}$ a $15^{\circ} 59^{\prime} \mathrm{S}$ e $47^{\circ} 55^{\prime}$ a $\left.47^{\circ} 58^{\prime} \mathrm{W}\right)$, situada ao sul do Distrito Federal, de propriedade da Universidade de Brasília e área nuclear da Reserva da Biosfera do Cerrado. O campo limpo úmido estudado ocupa uma área de 16 ha próxima à mata de galeria do córrego Taquara $\left(15^{\circ} 55^{\prime} 35,4^{\prime \prime}\right.$ a $15^{\circ} 56^{\prime} 4,1^{\prime \prime} \mathrm{S}$ e $47^{\circ} 54^{\prime} 20,8^{\prime}$ " a $47^{\circ} 54^{\prime} 21,9^{\prime \prime} \mathrm{W}$ ). A área de estudo e as áreas vizinhas de mata de galeria, de campo sujo e de cerrado sensu stricto sofreram uma queimada acidental na primeira semana de agosto de 1999, cerca de um mês antes do início deste trabalho.

O campo limpo estudado pertence à classe de solo hidromórfico com lençol freático superficial, com alagamento permanente em algumas depressões e temporário na estação chuvosa no restante da área. O clima da região é do tipo Aw, segundo a classificação de Köppen e caracteriza-se por duas estações bem definidas: uma quente e chuvosa (de outubro a abril) e outra fria e seca (de maio a setembro). A temperatura média anual máxima é de $28,5^{\circ} \mathrm{C}$ e a média anual mínima de $12,0{ }^{\circ} \mathrm{C}$. A precipitação média anual no período estudado foi de $1500 \mathrm{~mm}$, medida na estação meteorológica da Reserva Ecológica do IBGE.

\section{Amostragem}

Material botânico fértil foi coletado, quinzenalmente, de setembro de 1999 a fevereiro de 2001 no campo limpo úmido da FAL, ao longo de trilhas marcadas no sentido paralelo e perpendicular à borda da mata de galeria do córrego Taquara, de modo a percorrer a maior extensão possível da área.

As espécies foram previamente identificadas por meio de literatura especializada, comparação com material depositado no herbário da Universidade de Brasília (UB) e da Reserva Ecológica do Instituto Brasileiro de Geografia e Estatística (IBGE) e posteriormente por especialistas nos grupos taxonômicos. Os exemplares coletados foram herborizados de acordo com os procedimentos usuais e encontram-se depositados nos herbários anteriormente citados.

As espécies foram classificadas em famílias de acordo com o sistema do Angiosperm Phylogeny Group (APG 2003). Através do material coletado durante os levantamentos florísticos foi elaborada uma listagem contendo as famílias, gêneros e espécies e o número do coletor. Os nomes das espécies e de seus autores foram conferidos através de consultas ao The Plant Names Project (2000) e ao W³ Trópicos (http://www.mobot.org, março 2007).

Os resultados florísticos encontrados no campo limpo úmido da FAL foram comparados com outros trabalhos que contemplaram o estrato herbáceo-arbustivo de diferentes fitofisionomias do bioma Cerrado, desde ambientes úmidos, campo limpo úmido e vereda até campo sujo, cerrado sensu stricto e cerradão (Tabela 1) e que realizaram pelo menos um ano de levantamento, com coletas sistemáticas quinzenais ou mensais. Foram incluídas somente espécies herbáceas, subarbustivas, arbustivas e trepadeiras não lenhosas. Fizeram parte da composição da matriz 1.024 espécies. Para evitar o falso aumento das espécies e a falsa diferença florística entre as áreas devidas à incorreção no uso de nomes, a sinonímia e a grafia dos taxa foi conferida através do The Plant Names Project (2000) e da página W³ Trópicos 
Tabela 1. Trabalhos consultados para a comparação florística dos componentes herbáceo-arbustivos e arbustivos de diferentes fitofisionomias de Cerrado com o presente estudo. $\mathrm{N}$ = Número de espécies herbáceas, subarbustivas e arbustivas utilizadas na matriz de comparação.

Table 1. Papers consulted for floristic comparison of the herbaceous and shrub layer of different Cerrado physiognomies and the present study. $\mathrm{N}=\mathrm{Number}$ of herbaceous, subshrub and shrub species used in the comparison matrix.

\begin{tabular}{|c|c|c|c|c|c|}
\hline Referência & Local & Coordenadas & $\begin{array}{c}\text { Tamanho } \\
\text { das áreas } \\
\text { amostrada (ha) }\end{array}$ & Fitofisionomias & $\mathbf{N}$ \\
\hline $\begin{array}{l}\text { Silva \& Nogueira } \\
\text { (1999) }\end{array}$ & $\begin{array}{l}\text { Reserva Ecológica do } \\
\text { IBGE, DF }\end{array}$ & $15^{\circ} 55^{\prime} \mathrm{S}$ e $47^{\circ} 53^{\prime} \mathrm{W}$ & 300 & cerrado sensu stricto & 144 \\
\hline $\begin{array}{l}\text { Batalha \& Mantovani } \\
\text { (2001) }\end{array}$ & $\begin{array}{l}\text { Reserva Pé-de-Gigante, } \\
\text { SP }\end{array}$ & $\begin{array}{l}21^{\circ} 36-38^{\prime} \mathrm{S} \mathrm{e} \\
47^{\circ} 36-39^{\prime} \mathrm{W}\end{array}$ & $\begin{array}{l}135,9 ; 967,7 \\
96,8\end{array}$ & $\begin{array}{l}\text { cerradão, cerrado sensu } \\
\text { stricto, campo cerrado }\end{array}$ & $\begin{array}{l}70,181 \\
171\end{array}$ \\
\hline Araújo et al. (2002) & Uberlândia, MG & $\begin{array}{l}18^{\circ} 60^{\prime} \mathrm{S} \text { e } 48^{\circ} 18^{\prime} \mathrm{W} \\
19^{\circ} 03^{\prime} \mathrm{S} \text { e } 48^{\circ} 21^{\prime} \mathrm{W} \\
19^{\circ} 11^{\prime} \mathrm{S} \text { e } 48^{\circ} 24^{\prime} \mathrm{W}\end{array}$ & $45,8,5$ e 12 & Veredas $1,2,3$ e $4 * *$ & $\begin{array}{l}261,214 \\
182,196\end{array}$ \\
\hline $\begin{array}{l}\text { Tannus \& Assis } \\
(2004)\end{array}$ & Itirapina, SP & $\begin{array}{l}22^{\circ} 15^{\prime} 43^{\prime \prime}-00^{\prime \prime} \mathrm{S} \mathrm{e} \\
47^{\circ} 53^{\prime}-54^{\prime} \mathrm{W}\end{array}$ & $65^{*}$ & $\begin{array}{l}\text { campo sujo, campo } \\
\text { limpo úmido }\end{array}$ & 177,105 \\
\hline $\begin{array}{l}\text { Munhoz \& Felfili } \\
(2004)\end{array}$ & $\begin{array}{l}\text { Fazenda Água Limpa, } \\
\text { DF }\end{array}$ & $\begin{array}{l}15^{\circ} 56^{\prime}-15^{\circ} 59^{\prime} \mathrm{S} \text { e } \\
47^{\circ} 55^{\prime}-47^{\circ} 58^{\prime} \mathrm{W}\end{array}$ & 16 & campo sujo & 224 \\
\hline $\begin{array}{l}\text { Munhoz \& Felfili } \\
\text { (2006) }\end{array}$ & $\begin{array}{l}\text { Alto Paraíso de Goiás, } \\
\text { GO }\end{array}$ & $\begin{array}{l}14^{\circ} 04^{\prime} 8,83^{\prime \prime} \mathrm{S} \mathrm{e} \\
47^{\circ} 30^{\prime} 33,1^{\prime \prime} \mathrm{W}\end{array}$ & 21 & campo limpo úmido & 183 \\
\hline Presente estudo & $\begin{array}{l}\text { Fazenda Água Limpa, } \\
\text { DF }\end{array}$ & $\begin{array}{l}15^{\circ} 56^{\prime}-15^{\circ} 59^{\prime} \mathrm{S} \text { e } \\
47^{\circ} 55^{\prime}-47^{\circ} 58^{\prime} \mathrm{W}\end{array}$ & 16 & campo sujo & 224 \\
\hline
\end{tabular}

*As duas áreas totalizam 65 ha. ** Veredas 3 e 4 encontram-se na mesma localidade.

* The two sites totalize 65 ha. ** Palm swamp 3 and 4 situated in the same locality.

(http://www.mobot.org 2007). Espécies identificadas somente até o nível de gênero e família foram excluídas. Também não fizeram parte da composição da matriz de dados as espécies invasoras, segundo Mendonça et al. (1998) e os autores dos trabalhos consultados, e espécies com necessidade de confirmação da identificação (cf), a não ser que o gênero fosse citado apenas para uma das áreas.

Para avaliar a similaridade entre os componentes herbáceos, subarbustivos e arbustivos do campo limpo úmido da FAL com o das outras áreas utilizou-se o índice de similaridade de Sørensen, baseado na presença e ausência de espécies (Mueller-Dombois \& Ellenberg 1974), calculado utilizando-se o programa MVSP (Kovach Computing Services 2005).

Para a análise das relações florísticas entre as áreas, foi empregado Análise de Correspondência Retificada (DCA), realizada pelo programa PC-ORD versão 4.2 (McCune \& Mefford 1999), a partir de uma matriz de presença e ausência de espécies.

\section{Resultados}

Um total de 529 espécimes em fase reprodutiva, pertencentes a 197 espécies ou taxa infra-específicos, distribuídas em 106 gêneros e 41 famílias (Tabela 2), foram coletados no campo limpo úmido da FAL. Das 197 espécies, 183 foram identificadas até o nível de espécie, 14 ao nível de gênero, incluindo uma aparentemente nova para a ciência que se encontra em fase de descrição pelo taxonomista, Syngonanthus sp.1 (Eriocaulaceae). Três espécies foram determinadas somente até o nível de família e podem, também, vir a serem novas para a ciência.

Cinco espécies invasoras à flora do Cerrado foram registradas na área: Andropogon bicornis L., Andropogon leucostachyus Kunth, Borreria latifolia (Aubl.) K. Schum (ambas originárias das Américas do Sul e Central), Bulbostylis capillaris (L.) C.B. Clarke (originária da América do Norte) e Melinis repens (Willd.) Zizka (gramínea de origem africana) (Tabela 2).
Oito famílias contiveram $68,2 \%$ do total de espécies coletadas no campo limpo úmido da FAL: Poaceae (39 spp.); Cyperaceae (24 spp.); Asteraceae (21 spp.); Polygalaceae (15 spp.); Eriocaulaceae (12 spp.); Xyridaceae e Melastomataceae ( 8 spp. cada) e Fabaceae ( 7 spp.). Por outro lado, 15 (37,5\%) famílias apresentaram uma única espécie, seis $(15,0 \%)$ apresentaram duas espécies e outras cinco $(12,5 \%)$ apresentaram três espécies.

A maioria dos gêneros amostrados $(75,1 \%)$ apresentou uma única espécie, sugerindo também uma alta diversidade genérica nessa fisionomia. Os seis gêneros com maior número de espécies, e que compreendem $29,9 \%$ do total de espécies amostradas, foram Rhynchospora (15 spp.); Paspalum (13 spp.); Polygala (12 spp.); Xyris (7 spp.) e Andropogon e Syngonanthus (6 spp. cada).

O hábito herbáceo foi predominante no campo limpo úmido estudado, com 139 (70,2\%) espécies, seguido pelo subarbustivo com $42(21,2 \%)$ espécies e do arbustivo com 14 (7,0\%) espécies, apenas três espécies de trepadeiras foram amostradas, correspondendo a $1,5 \%$ do total de espécies.

Os valores de similaridade florística para o índice de Sørensen entre o campo limpo úmido estudado e as outras 12 áreas de Cerrado variaram de 3,3 a 37,4\%. O campo limpo úmido da FAL foi mais similar ao campo sujo localizado na FAL, que lhe é contíguo, seguido das áreas de campo limpo úmido de Alto Paraíso de Goiás e de Veredas de Uberlândia, MG. Os valores mais baixos foram observados em relação ao estrato herbáceo-arbustivo do Cerradão, do cerrado sensu stricto, do campo cerrado e ao campo sujo situados no Estado de São Paulo (Reserva Pé-de-Gigante e Município de Ipiranga). Nenhuma das espécies encontradas no presente estudo ocorreu em todas as outras áreas comparadas. As espécies mais freqüentes foram Echinolaena inflexa (observada em $92 \%$ das áreas), Andropogon virgatus e Trimezia juncifolia (61\%), Bulbostylis junciformis, Cuphea linarioides, Curtia tenuifolia, Ludwigia nervosa, Paspalum gardnerianum, Polygala longicaulis, Rhynchospora globosa, $R$. rugosa e Tibouchina gracilis (53\%). 
Tabela 2. Espécies da flora vascular, em ordem de família, em um campo limpo úmido na Fazenda Água Limpa, Brasília, DF. N = Número do coletor C. Munhoz;

Table 2. Species of the vascular flora, ordered by family, in a moist grassland in Água Limpa Farm, Brasília, DF. N = Collector number C. Munhoz;

\begin{tabular}{|c|c|c|}
\hline Família/Espécie & Hábito & $\mathbf{N}$ \\
\hline \multicolumn{3}{|l|}{ ACANTHACEAE } \\
\hline Justicia pycnophylla Lindau & Subarbusto & 916 \\
\hline Ruellia incompta (Nees) Lindau & Subarbusto & 1373 \\
\hline \multicolumn{3}{|l|}{ ALSTROEMERIACEAE } \\
\hline Alstroemeria longistyla Schenk & Erva & 956 \\
\hline \multicolumn{3}{|l|}{ AMARANTHACEAE } \\
\hline Pfaffia jubata Mart. & Subarbusto & 890 \\
\hline Xerosiphon aphylla (Pohl ex Moq.) Pedersen & Erva & 741 \\
\hline Amaranthaceae & Subarbusto & 2385 \\
\hline \multicolumn{3}{|l|}{ APIACEAE } \\
\hline Eryngium marginatum Pohl ex Urb. & Erva & 812 \\
\hline Eryngium sp. & Erva & 2096 \\
\hline \multicolumn{3}{|l|}{ APOCYNACEAE } \\
\hline Asclepias candida Vell. & Subarbusto & 794 \\
\hline Ditassa cordata (Turcz.) Fontella & Erva & 1320 \\
\hline Mandevilla rugosa (Benth.) Woodson & Subarbusto & 961 \\
\hline Oxypetalum aequaliflorum E. Fourn. & Erva & 786 \\
\hline \multicolumn{3}{|l|}{ ASTERACEAE } \\
\hline Achyrocline alata (Kunth) DC. & Erva & 1187 \\
\hline Aspilia foliacea (Spreng.) Baker & Subarbusto & 2454 \\
\hline Baccharis erigeroides DC. & Subarbusto & 2013 \\
\hline Baccharis humilis Sch. Bip.ex Baker & Erva & 734 \\
\hline Baccharis subdentata DC. & Erva & 959 \\
\hline Calea gardneriana Baker & Erva & 2034 \\
\hline Calea platylepis Sch. Bip.ex Baker & Subarbusto & 2011 \\
\hline Chromolaena vindex (DC.) R.M.King \& H.Rob. & Erva & 740 \\
\hline Elephantopus elongatus Gardner & Subarbusto & 965 \\
\hline Ichthyothere latifolia Baker & Subarbusto & 2009 \\
\hline Lessingianthus desertorum (Mart. ex DC.) H.Rob. & Erva & 1859 \\
\hline Lessingianthus grearii (H.Rob.) H.Rob. & Subarbusto & 889 \\
\hline Lessingianthus simplex (Less.) H.Rob. & Erva & 1864 \\
\hline Mikania officinalis Mart. & Erva & 2402 \\
\hline Riencourtia oblongifolia Gardner & Arbusto & 2392 \\
\hline Stevia heptachaeta DC. & Erva & 1497 \\
\hline Trichogonia sp. & Subarbusto & 986 \\
\hline Vernonia foliosa Gardner & Subarbusto & 1697 \\
\hline Wedelia bishopii H. Rob. & Subarbusto & 1456 \\
\hline Asteraceae 1 & Arbusto & 2529 \\
\hline Asteraceae 2 & Subarbusto & 2032 \\
\hline \multicolumn{3}{|l|}{ BORAGINACEAE } \\
\hline Cordia calocephala Cham. & Subarbusto & 2395 \\
\hline \multicolumn{3}{|l|}{ BURMANIACEAE } \\
\hline Burmannia flava Mart. & Erva & 1003 \\
\hline \multicolumn{3}{|l|}{ CAMPANULACEAE } \\
\hline Lobelia camporum Pohl & Erva & 2439 \\
\hline \multicolumn{3}{|l|}{ CHRYSOBALANACEAE } \\
\hline Parinari obtusifolia Hook. f. & Arbusto & 886 \\
\hline
\end{tabular}


Tabela 2. Continuação...

\begin{tabular}{|c|c|c|}
\hline Família/Espécie & Hábito & $\mathbf{N}$ \\
\hline \multicolumn{3}{|l|}{ CONVOLVULACEAE } \\
\hline Ipomoea procurrens Meisn. & Trepadeira & 2219 \\
\hline \multicolumn{3}{|l|}{ CYPERACEAE } \\
\hline Ascolepis brasiliensis (Kunth) Benth. ex C.B. Clarke & Erva & 2039 \\
\hline Bulbostylis capillaris (L.) C.B. Clarke* & Erva & 1191 \\
\hline Bulbostylis junciformis (Kunth) C.B. Clarke & Erva & 1323 \\
\hline Bulbostylis paraensis C.B. Clarke & Erva & 828 \\
\hline Bulbostylis sellowiana (Kunth) Palla & Erva & 905 \\
\hline Lagenocarpus rigidus (Kunth) Nees & Erva & 2214 \\
\hline Rhynchospora cf. albiceps Kunth & Erva & 888 \\
\hline Rhynchospora brasiliensis Boeck. & Erva & 948 \\
\hline Rhynchospora consanguinea (Kunth) Boeck. & Erva & 2129 \\
\hline Rhynchospora emaciata (Nees) Boeck. & Erva & 1181 \\
\hline Rhynchospora gigantea Link & Erva & 1293 \\
\hline Rhynchospora globosa (Kunth) Roem. \& Schult. & Erva & 1324 \\
\hline Rhynchospora graminea Uittien & Erva & 899 \\
\hline Rhynchospora marisculus Nees & Erva & 899 \\
\hline Rhynchospora robusta (Kunth) Boeck. & Erva & 2398 \\
\hline Rhynchospora rugosa (Vahl) Gale & Erva & 1006 \\
\hline Rhynchospora tenuis Link & Erva & 2106 \\
\hline Rhynchospora velutina (Kunth) Boeck. & Erva & 904 \\
\hline Rhynchospora sp.1 & Erva & 826 \\
\hline Rhynchospora sp.2 & Erva & 1594 \\
\hline Rhynchospora sp.3 & Erva & 2212 \\
\hline Scleria hirtella Sw. & Erva & 894 \\
\hline Scleria leptostachya Kunth & Erva & 2522 \\
\hline Scleria sp. & Erva & 898 \\
\hline \multicolumn{3}{|l|}{ DROSERACEAE } \\
\hline Drosera montana A. St.-Hil. & Erva & - \\
\hline \multicolumn{3}{|l|}{ ERIOCAULACEAE } \\
\hline Eriocaulon modestum Kunth & Erva & 1584 \\
\hline Paepalanthus eriocauloides Ruhland & Erva & 1480 \\
\hline Paepalanthus flaccidus (Bong.) Kunth & Erva & 1183 \\
\hline Paepalanthus lundii Körn. & Erva & 1858 \\
\hline Paepalanthus cf. speciosus Gardner & Erva & 1599 \\
\hline Syngonanthus densiflorus (Körn.) Ruhland & Erva & 832 \\
\hline Syngonanthus gracilis (Bong.) Ruhland & Erva & 1707 \\
\hline Syngonanthus nitens (Bong.) Ruhl. & Erva & 1186 \\
\hline Syngonanthus xeranthemoides (Bong.) Ruhland & Erva & 886 \\
\hline Syngonanthus sp.1 ** & Erva & 1817 \\
\hline Syngonanthus sp.2 & Erva & 1580 \\
\hline \multicolumn{3}{|l|}{ ERYTHROXYLACEAE } \\
\hline Erythroxylum nanum A. St.-Hil. & Subarbusto & 2008 \\
\hline \multicolumn{3}{|l|}{ EUPHORBIACEAE } \\
\hline Acalypha clausenii (Turcz.) Müll. Arg. & Erva & 1961 \\
\hline Chamaesyce caecorum (Mart. ex Boiss.) Croizat & Erva & 1868 \\
\hline Croton antisyphiliticus Mart. & Subarbusto & 2022 \\
\hline Dalechampia caperonioides Baill. & Erva & 1866 \\
\hline
\end{tabular}


Tabela 2. Continuação...

\begin{tabular}{|c|c|c|}
\hline Família/Espécie & Hábito & $\mathbf{N}$ \\
\hline \multicolumn{3}{|l|}{ FABACEAE } \\
\hline Chamaecrista $\mathrm{cf}$. pohliana (Benth.) H.S. Irwin \& Barneby & Subarbusto & 2144 \\
\hline Chamaecrista conferta Benth. & Subarbusto & $1176 \mathrm{a}$ \\
\hline Crotalaria cf. goiasensis Windler \& S. Skinner & Subarbusto & 2535 \\
\hline Eriosema defoliatum Benth. & Subarbusto & 1962 \\
\hline Galactia grewiifolia (Benth.) Taub. & Subarbusto & 1959 \\
\hline Mimosa setosa Benth. & Arbusto & 2143 \\
\hline Vigna cf. linearis (Kunth) Maréchal, Mascherpa \& Stainier & Trepadeira & 1000 \\
\hline \multicolumn{3}{|l|}{ GENTIANACEAE } \\
\hline Curtia tenuifolia (Aubl.) Knobl. & Erva & 967 \\
\hline Deianira chiquitana Herzog & Erva & 1482 \\
\hline Schultesia gracilis Mart. & Erva & 993 \\
\hline \multicolumn{3}{|l|}{ GESNERIACEAE } \\
\hline Sinningia elatior (Kunth)Chautems & Subarbusto & 2534 \\
\hline \multicolumn{3}{|l|}{ IRIDACEAE } \\
\hline Sisyrinchium restioides Spreng. & Erva & 1493 \\
\hline Sisyrinchium vaginatum Spreng. & Erva & 2525 \\
\hline Trimezia juncifolia (Klatt.) Benth. \& Hook. f. & Erva & 1121 \\
\hline \multicolumn{3}{|l|}{ LAMIACEAE } \\
\hline Hyptis carpinifolia Benth. & Erva & 964 \\
\hline Hyptis linarioides Pohl ex Benth. & Subarbusto & 1865 \\
\hline Hyptis subrotunda Pohl ex Benth. & Arbusto & 1299 \\
\hline Rhabdocaulon denudatum (Benth.) Epling & Erva & 1856 \\
\hline \multicolumn{3}{|l|}{ LENTIBULARIACEAE } \\
\hline Utricularia amethystina Salzm. ex A. St.-Hil. \& Girard & Erva & 998 \\
\hline \multicolumn{3}{|l|}{ LYTHRACEAE } \\
\hline Cuphea linarioides Cham. \& Schltdl. & Subarbusto & 2001 \\
\hline Cuphea spermacoce A. St.-Hil. & Subarbusto & 2000 \\
\hline Diplusodon virgatus Pohl & Arbusto & 1302 \\
\hline \multicolumn{3}{|l|}{ MALPIGHIACEAE } \\
\hline Banisteriopsis megaphylla (A. Juss.) B. Gates & Trepadeira & 2437 \\
\hline Tetrapterys ambigua (A. Juss.) Nied. & Subarbusto & 2003 \\
\hline \multicolumn{3}{|l|}{ MALVACEAE } \\
\hline Sida cf. linearifolia A. St.-Hil. & Erva & 1045 \\
\hline \multicolumn{3}{|l|}{ MELASTOMATACEAE } \\
\hline Clidemia capitellata (Bonpl.) D. Don & Arbusto & - \\
\hline Comolia lanceaeflora Triana & Subarbusto & 1297 \\
\hline Desmocelis villosa (Aubl.) Naudin & Subarbusto & - \\
\hline Leandra polystachya (Naudin) Cogn. & Arbusto & 1999 \\
\hline Microlicia polystemma Naudin & Subarbusto & 2527 \\
\hline Rhynchanthera grandiflora (Aubl.) DC. & Subarbusto & 1491 \\
\hline Tibouchina aegopogon (Naudin) Cogn. & Arbusto & 1301 \\
\hline Tibouchina gracilis (Bonpl.) Cogn. & Arbusto & 960 \\
\hline \multicolumn{3}{|l|}{ MENISPERMACEAE } \\
\hline Cissampelos ovalifolia DC. & Subarbusto & 2453 \\
\hline \multicolumn{3}{|l|}{ MYRTACEAE } \\
\hline Eugenia bracteata Vell. & Subarbusto & 2123 \\
\hline Myrcia decrescens O. Berg. & Erva & 1855 \\
\hline
\end{tabular}


Tabela 2. Continuação...

\begin{tabular}{|c|c|c|}
\hline Família/Espécie & Hábito & $\mathbf{N}$ \\
\hline Myrcia torta DC. & Arbusto & 2041 \\
\hline \multicolumn{3}{|l|}{ OCHNACEAE } \\
\hline Sauvagesia linearifolia A. St.-Hil. & Erva & 1478 \\
\hline Sauvagesia racemosa A. St.-Hil. & Subarbusto & 2275 \\
\hline \multicolumn{3}{|l|}{ ONAGRACEAE } \\
\hline Ludwigia nervosa (Poir.) Hara & Arbusto & 1712 \\
\hline \multicolumn{3}{|l|}{ ORCHIDACEAE } \\
\hline Cleistes sp. & Erva & 2213 \\
\hline Habenaria nasuta Rchb. f. \& Warm. & Erva & 989 \\
\hline Habenaria cf. urbaniana Cogn. & Erva & 1495 \\
\hline \multicolumn{3}{|l|}{ OROBANCHACEAE } \\
\hline Alectra sp. & Erva & 1575 \\
\hline Buchnera lavandulacea Cham. \& Schldl. & Erva & 906 \\
\hline \multicolumn{3}{|l|}{ OXALIDACEAE } \\
\hline Oxalis densifolia Mart. \& Zucc. & Erva & 2138 \\
\hline \multicolumn{3}{|l|}{ POACEAE } \\
\hline Andropogon bicornis L.* & Erva & 2016 \\
\hline Andropogon lateralis Nees & Erva & 2025 \\
\hline Andropogon lateralis subsp. cryptopus (Hack.) A. Zanin & Erva & 754 \\
\hline Andropogon leucostachyus Kunth* & Erva & 758 \\
\hline Andropogon selloanus (Hack.) Hack. & Erva & 796 \\
\hline Andropogon virgatus Desv. ex Dandy & Erva & 935 \\
\hline Arthropogon filifolius Filg. & Erva & 743 \\
\hline Arthropogon villosus Nees & Erva & 749 \\
\hline Arundinella hispida (Humb. \& Bonpl. Ex Willd.) Kuntze & Erva & 1201 \\
\hline Axonopus brasiliensis (Spreng.) Kuhlm. & Erva & 2028 \\
\hline Axonopus comans (Trin.) Henrard & Erva & 752 \\
\hline Axonopus sp. & Erva & 2142 \\
\hline Ctenium cf. brachystachyum (Nees) Kunth & Erva & 742 \\
\hline Ctenium cirrhosum (Nees) Kunth & Erva & 1333 \\
\hline Echinolaena inflexa (Poir.) Chase & Erva & - \\
\hline Elionurus muticus (Spreng.) Kuntze & Erva & 2024 \\
\hline Hyparrhenia bracteata (Humb. \& Bonpl. ex Willd.) Stapf & Erva & 2186 \\
\hline Lasiacis standleyi Hitchc. & Erva & 1969 \\
\hline Melinis repens (Willd.) Zizka.* & Erva & 976 \\
\hline Mesosetum ferrugineum (Trin.) Chase & Erva & 757 \\
\hline Otachyrium seminudum Hack. ex Send. \& Soderstr. & Erva & 900 \\
\hline Panicum cyanescens Nees ex Trin. & Erva & 2533 \\
\hline Panicum parvifolium Lam. & Erva & 901 \\
\hline Paspalum dedeccae Quarin & Erva & 2027 \\
\hline Paspalum ellipticum Döll & Erva & 751 \\
\hline Paspalum erianthum Nees ex Trin. & Erva & 1960 \\
\hline Paspalum gardnerianum Nees & Erva & 1869 \\
\hline Paspalum geminiflorum Steud. & Erva & 2271 \\
\hline Paspalum glaucescens Hack. & Erva & 988 \\
\hline Paspalum hyalinum Nees ex Trin. & Erva & 1326 \\
\hline Paspalum imbricatum Filg. & Erva & 941 \\
\hline Paspalum lineare Trin. & Erva & - \\
\hline Paspalum maculosum Trin. & Erva & 937 \\
\hline
\end{tabular}


Tabela 2. Continuação...

\begin{tabular}{|c|c|c|}
\hline Família/Espécie & Hábito & $\mathbf{N}$ \\
\hline Paspalum pectinatum Nees ex Trin. & Erva & 2030 \\
\hline Paspalum polyphyllum Nees ex Trin. & Erva & 2040 \\
\hline Paspalum stellatum Humb. \& Bonpl. in Flüeggé & Erva & 1814 \\
\hline Schizachyrium cf. condensatum (Kunth) Nees & Erva & 2456 \\
\hline Schizachyrium sp. & Erva & 1214 \\
\hline Sporobulus reflexus Boechat \& Longhi-Wagner & Erva & 738 \\
\hline \multicolumn{3}{|l|}{ POLYGALACEAE } \\
\hline Monnina exaltata A.W. Benn. & Erva & 907 \\
\hline Monnina martiana Klotzsch ex A.W. Benn. & Arbusto & 2021 \\
\hline Monnina stenophylla A. St.-Hil. & Erva & 1966 \\
\hline Polygala carphoides Chodat & Erva & 953 \\
\hline Polygala cf. exigua A.W. Benn & Erva & 2442 \\
\hline Polygala galioides Poir. & Erva & 908 \\
\hline Polygala gracilis Kunth & Erva & 1199 \\
\hline Polygala harleyi M.C.M. Marques & Erva & $1177 \mathrm{a}$ \\
\hline Polygala hygrophila Humb., Bonpl. \& Kunth & Erva & 963 \\
\hline Polygala longicaulis Humb., Bonpl. \& Kunth & Erva & 1211 \\
\hline Polygala misella Bernardi & Erva & 1004 \\
\hline Polygala monosperma A.W. Benn. & Erva & 795 \\
\hline Polygala subtilis Humb., Bonpl. \& Kunth & Erva & 972 \\
\hline Polygala tenuis DC. & Erva & 1850 \\
\hline Polygala timoutou Aubl. & Erva & 1314 \\
\hline \multicolumn{3}{|l|}{ RUBIACEAE } \\
\hline Borreria irwiniana E. L. Cabral & Erva & $1127 \mathrm{~A}$ \\
\hline Borreria latifolia ((Aubl.) K. Schum.* & Erva & 1193 \\
\hline Borreria poaya (A. St.-Hil.) DC. & Erva & 2124 \\
\hline Borreria marticrovettiana E. L. Cabral & Subarbusto & 1002 \\
\hline Borreria tenella Cham. \& Schltdl. & Subarbusto & 1194 \\
\hline \multicolumn{3}{|l|}{ SANTALACEAE } \\
\hline Thesium brasiliense A. DC. & Erva & 974 \\
\hline \multicolumn{3}{|l|}{ SOLANACEAE } \\
\hline Solanum subumbellatum Vell. & Subarbusto & 2015 \\
\hline \multicolumn{3}{|l|}{ TURNERACEAE } \\
\hline Piriqueta sidifolia Urb. & Subarbusto & 1933 \\
\hline Turnera oblongifolia Cambess. & Subarbusto & 2098 \\
\hline \multicolumn{3}{|l|}{ VERBENACEAE } \\
\hline Lippia corymbosa Cham. & Arbusto & 1587 \\
\hline \multicolumn{3}{|l|}{ XYRIDACEAE } \\
\hline Abolboda poarchon Seub. & Erva & 829 \\
\hline Xyris guaranitica Malme & Erva & 891 \\
\hline Xyris hymenachne Mart. & Erva & 1592 \\
\hline Xyris jupicai Rich. & Erva & 2288 \\
\hline Xyris schizachne Mart. & Erva & 1714 \\
\hline Xyris tortula Mart. & Erva & 2289 \\
\hline Xyris sp.1 & Erva & 827 \\
\hline Xyris sp.2 & Erva & 2044 \\
\hline
\end{tabular}

* = espécie invasora; ** = espécie nova segundo especialista no grupo taxonômico; espécies em negrito = nomes não citados em Proença et al. (2001) para a flora do Distrito Federal.

$*=$ weedy exotic species; $* *=$ a probably new species according to a taxonomist of the group; bold = names not listed by Proença et al. $(2001)$ for the flora of Brazil Federal District. 
A ordenação por DCA evidenciou no primeiro eixo um gradiente contínuo de mudanças de composição florística, onde as fitofisionomias mais úmidas estão à esquerda e as mais secas progressivamente à direita (Figura 1). O primeiro eixo da ordenação mostra um gradiente geográfico no sentido do Centro-oeste para o Sudeste de áreas de vegetação lenhosa esparsa (campo sujo) para as mais densas (campo cerrado até cerradão). Esta variabilidade entre as áreas secas ao longo do eixo 1 foi tão grande quanto para as úmidas no segundo eixo (autovalores variando, aproximadamente de 10 a 60).

\section{Discussão}

Apesar da sua pequena extensão, o campo limpo úmido da FAL apresentou uma grande riqueza florística. Dos 197 taxa registrados, 28 (14,6\%) espécies não estão relacionados na lista de espécies elaborada para o Distrito Federal por Proença et al. (2001). Foi registrada a ocorrência de uma espécie desconhecida pela ciência, e algumas das espécies não identificadas até o nível de espécie nesse trabalho, podem, também, vir a ser novas para a ciência. Inventários florísticos nas fisionomias de Cerrado têm revelado espécies novas para a ciência (Batalha \& Martins 2002), e vêm contribuindo com adições à listagem da flora vascular (Mendonça et al. 1998), sugerindo que esse bioma não foi ainda satisfatoriamente coletado. Prance et al. (2000) ressaltam que a flora tropical de um modo geral ainda carece de um grande esforço de coleta.

A diversidade alfa da camada herbácea de Cerrado das fitofisionomias de todos os trabalhos analisados foi alta, variando de 70 a 261 espécies. O número de espécies amostradas na FAL foi superior ao observado em uma área de campo limpo úmido em Itirapina

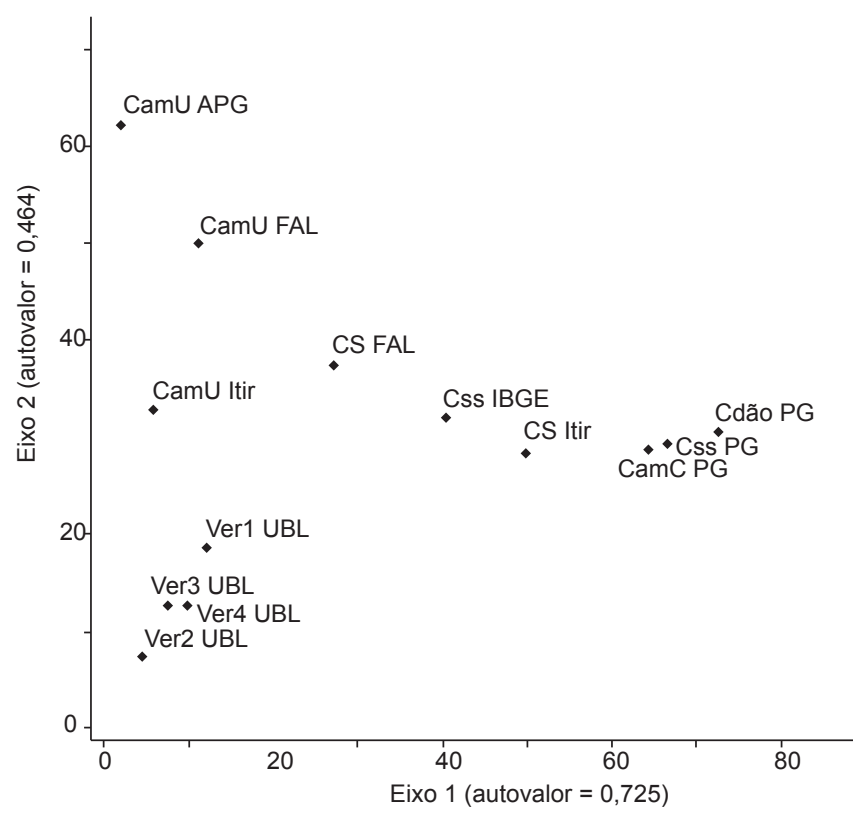

Figura 1. Posicionamento por eixos de ordenação (DCA) dos componentes herbáceo-subarbustivos e arbustivos de 13 áreas, em diferentes fitofisionomias de Cerrado. Cdão = Cerradão; Css = Cerrado sensu stricto; CamC = Campo cerrado; $\mathrm{CS}=$ Campo sujo; $\mathrm{CamU}=\mathrm{Campo}$ limpo úmido; Ver = Vereda PG = eserva Pé-de-Gigante, $\mathrm{SP} ; \mathrm{FAL}=$ Fazenda Água Limpa, DF; APG = Alto Paraíso de Goiás-GO, Itir = Itirapina, SP e UBL = Uberlândia, MG.

Figure 1. Positioning in the DCA ordination axes of the herbaceous and subshrub layer of 13 sites in Brazilian Cerrado physiognomies. Cdão = Cerradão; Css = Cerrado sensu stricto; CamC = Campo cerrado; $\mathrm{CS}=$ Campo sujo; CamU = Campo limpo úmido; Ver = palm swamp, Uberlândia, $\mathrm{MG}$; PG = Péde-Gigante Reserve, SP; FAL = Água Limpa Farm, DF; APG = Alto Paraíso de Goiás, GO and Itir = Itirapina, SP.
(SP) (Tannus \& Assis 2004), similar ao encontrado em Alto Paraíso de Goiás (GO) (Munhoz \& Felfili 2006a) e inferior à flora de veredas amostradas em Uberlândia (MG) (Araújo et al. 2002), possivelmente por diferenças nos tamanhos entre as áreas amostradas, na matriz vegetacional onde as mesmas estão inseridas e pelo aumento de espécies subarbustivas e arbustivas nas áreas mais drenadas das veredas. Munhoz \& Felfili (2004) e Tannus \& Assis (2004) encontraram número superior de espécies herbáceo-subarbustivas e arbustivas em campo sujo do que em áreas de campo limpo úmido adjacentes, porém em áreas de cerrado sensu stricto o número de taxa neste componente foi menor que o das áreas úmidas (Mantovani \& Martins 1993, Silva \& Nogueira 1999, Batalha \& Mantovani 2001). Estas observações sugerem que para a camada rasteira, nas formações campestres úmidas o número de espécies de uma determinada área pode estar relacionado com um gradiente de umidade, com maior número de espécies nas porções mais drenadas e redução nas permanentemente alagadas. Já nas formações savânicas, a redução no número de espécies da camada herbáceo-subarbustiva, provavelmente se dá em consequiência do aumento de indivíduos arbustivos e arbóreos. Araújo et al. (2002) encontraram maior riqueza de espécies nas zonas bem drenadas de vereda do que nas alagadas, onde os autores observaram uma flora específica destes ambientes. Padrão semelhante tem sido encontrado em matas de galeria no Brasil Central, com maior número de espécies nas porções mais drenadas e menor nas encharcadas (Sampaio et al. 2000, Silva Jr. 2001). O aumento na riqueza de espécies, também coincidiu com a diminuição de água no solo e o aumento nas cotas altitudinais em dez fitofisionomias distintas no Pantanal (Pinder \& Rosso 1998).

Famílias como Poaceae, Cyperaceae, Asteraceae, Polygalaceae, Eriocaulaceae e Xyridaceae que apresentaram elevada riqueza na FAL, também tiveram alta riqueza em outras áreas úmidas de Cerrado (Araújo et al. 2002, Tannus \& Assis 2004, Munhoz \& Felfili 2006a). Destas, as três primeiras estão entre as mais ricas para a flora do Cerrado (s.l.) (Mendonça et al. 1998) e as três últimas mais associadas a áreas campestres úmidas ou a campos rupestres, principalmente Xyridaceae e Eriocaulaceae (Pirani et al. 1994), e pouco representadas ou ausentes em áreas de campo sujo (Munhoz \& Felfili 2004, Tannus \& Assis 2004) e de cerrado sensu stricto (Mantovani \& Martins 1993, Silva \& Nogueira 1999, Batalha \& Mantovani 2001, Batalha \& Martins 2002). Essas famílias foram responsáveis pela dominância do estrato herbáceo-subarbustivo na FAL, possivelmente devido às características edáficas, especialmente as condições de umidade, que dificultam o estabelecimento de indivíduos arbustivos e arbóreos.

A análise de similaridade evidenciou uma baixa sobreposição de espécies entre as 13 áreas comparadas neste estudo. Na família Poaceae, por exemplo, das 101 espécies que compuseram a matriz de análise, aproximadamente $36 \%$ ocorreram em apenas uma área. Somente uma espécie, Echinolaena inflexa ocorreu em 12 áreas, Andropogon virgatus, Axonopus siccus, Loudetiopsis chrysothrix e Schizachyrium condensatum, ocorreram em oito áreas, sendo a primeira mais associada às áreas campestres úmidas, e as três últimas não estiveram restritas a uma única formação, ocorrendo tanto em formações savânicas como em campestres úmidas ou secas. Algumas espécies, provavelmente adaptadas à saturação de água no solo, como, por exemplo, Curtia tenuifolia, Desmocelis villosa, Ludwigia nervosa, Paepalanthus flaccidus, Paspalum lineare, Polygala longicaulis, Rhynchospora albiceps, R. globosa, R. graminea, R. rugosa, Syngonanthus densiflorus e $S$. nitens foram encontradas somente em campo limpo úmido e em vereda sobre solos hidromórficos.

As baixas similaridades entre as áreas comparadas podem estar relacionadas diferenças na saturação hídrica do solo entre as áreas comparadas, à diferenças no tamanho das mesmas, ou porque muitas espécies têm distribuição esporádica ou em mosaicos (Castro et al. 
1999, Ratter et al. 2003). No entanto, segundo Chao et al. (2005, 2006), índices de similaridade baseados em incidências (presenças ou ausências) podem produzir estimativas desviadas, mesmo quando as amostragens são quase completas, pois se pode esperar que algumas espécies que estão presentes em uma área não são vistas por azares de amostragem. No caso de sub-amostragens e, em especial, no caso de comunidades com alta riqueza estes desvios são mais evidentes e podem até mudar as conclusões do estudo. Estes autores demonstraram que os desvios podem ser corrigidos, quando dados quantitativos são analisados, pois então é possível saber que proporções das espécies são raras e poderiam ter sido compartilhadas entre áreas, mas não o foram, pois embora estejam na área não foram detectadas na amostragem. Desta forma, os valores de similaridade apresentados neste estudo devem ser olhados com cautela, pois a aparente restrição das espécies amostradas uma única vez pode ser apenas devida a efeito de sub-amostragem.

A análise de ordenação por DCA demonstrou não haver uma uniformidade na composição florística entre as 13 áreas e evidenciou um gradiente contínuo de mudanças de composição florística das fitofisionomias mais úmidas para as mais secas. Entre as áreas úmidas, houve uma separação em um gradiente que vai dos campos úmidos para as veredas. A distância geográfica pode ter influenciado esta heterogeneidade florística no componente herbáceo-subarbustivo, visto que, as áreas analisadas em São Paulo, Minas Gerais, Goiás e no Distrito Federal, apresentam situações distintas de pluviosidade, altitude, bacias hidrográficas e domínio vegetacional. Para a flora herbáceo-subarbustiva de Cerrado, o endemismo das espécies pode, também, implicar na distribuição geográfica restrita de suas espécies, como encontrado no componente arbustivo-arbóreo (Castro et al. 1999).

O número de trabalhos comparados neste estudo está muito aquém do necessário para conclusões abrangentes sobre a distribuição das espécies do estrato herbáceo-arbustivo de Cerrado. No Distrito Federal, existem poucas áreas remanescentes de campo limpos úmidos e veredas, como se observa na região do Cerrado como um todo. No entanto, essas áreas, normalmente associadas com nascentes vêm sendo destruídas, sem que haja um pleno conhecimento dos seus recursos naturais e desses ecossistemas. A existência de uma flora de campo limpo úmido com grande riqueza reforça a necessidade da multiplicação desse tipo de investigação para outras áreas, ampliandose, assim, o conhecimento florístico dessa fitofisionomia e servindo como instrumento na avaliação e planejamento de ações de manejo, recuperação ambiental desses ambientes e criação de unidades de conservação que contemplem esta fitocenose.

\section{Agradecimentos}

As autoras agradecem à Kennya Mara Oliveira Ramos e ao Newton Rodrigues pelo apoio e auxílio no trabalho de campo; à CAPES pela bolsa de doutorado concedida para a primeira autora, ao DFID-UK, PRONEX-2 e CNPq-PELD pelo apoio financeiro que permitiu consolidar a infra-estrutura necessária para a execução deste projeto. À equipe técnica do herbário do IBGE, especialmente à Marina Fonseca e Roberta C. Mendonça. Pela identificação do material botânico, agradecemos aos taxonomistas: A. Goldberg (Turneraceae); A.O.S. Vieira (Campanulaceae); A. P. Prata (Cyperaceae); C. Anderson (Malpighiaceae); C. Fagg (Fabaceae); D.C.Wasshausen (Acanthaceae); E. Guimarães (Gentianaceae); E. L. Cabral (Rubiaceae); G.L. Webster (Euphorbiaceae); H. Robinson (Asteraceae); J.A. Lombardi (Vitaceae); J.N. Nakajima (Asteraceae); Josafa Carlos de Siqueira (Amaranthaceae); Katia Calago (Asteraceae); Luciano B. Biancheti (Orchidaceae); C. Proença (Myrtaceae e várias famílias); Mario Gomes (Rubiaceae); M. Magenta (Asteraceae); M.
C. Marques (Polygalaceae); M. G. L.Wanderley (Xyridaceae); N. Hensold (Eriocaulaceae); N. Marquete (Combretaceae); P.J.M. Maas (Burmaniaceae); L. Milhomem (Asteraceae); Ronaldo Marquete (Eriocaulaceae); R. Marquete (Asclepiadaceae e Gentianaceae); R. C. Oliveira (Poaceae); S.F. Smith (Ericaceae e Xyridaceae); V. L. Rivera \& L. Fonseca (Asteraceae); V. C. Souza (Scrophulariaceae); W.R. Anderson (Malpighiaceae); M. Simon (Fabaceae); T. Filgueiras (Poaceae).

\section{Referências Bibliográficas}

ANGIOSPERM PHYLOGENY GROUP. 2003. An update of the Angiosperm Phylogeny Group classification for the orders and families of flowering plants: APG II. Bot. J. Linn. Soc. 141(4):399-436.

ARAÚJO, G.M., BARBOSA, A.A.A., ARANTES, A.A. \& AMARAL, A.F. 2002. Composição florística de veredas no município de Uberlândia, MG. Rev. Brasil. Bot. 25(4):475-494.

BATALHA M.A. \& MANTOVANI, W. 2001. Floristic composition of the cerrado in the Pé-de-Gigante Reserve (Santa Rita do Passa Quatro, Southeastern Brazil). Acta Bot. Bras. 15(3):289-304.

BATALHA M.A. \& MARTINS F.R. 2002. The vascular flora of the cerrado in Emas National Park (central Brazil). Sida 20(1):295-312.

BATALHA, M.A. \& MARTINS, F.R. 2007. The vascular flora of the cerrado in Emas National Park (Central Brazil): a savanna flora summarized. Braz. Arch. Biol. Technol. 50(2):269-277.

CASTRO, A.A.J.F., MARTINS, F.R., TAMASHIRO, J.Y. \& SHEPHERD, G.J. 1999. How rich is the flora of Brazilian cerrados? Ann. Miss. Bot. Gard. 86(1):192-224.

CHAO, A., CHAZDON, R.L., COLWELL, R.K. \& SHEN, T.-J. 2005. A new statistical approach for assessing similarity of species composition with incidence and abundance data. Ecol. Lett. 8(2):148-159.

CHAO, A., CHAZDON, R.L., COLWELL, R.K. \& SHEN, T.-J. 2006. Abundance-based similarity indices and their estimation when there are unseen species in samples. Biometrics 62(2):361-371.

FELFILI, J., FILGUEIRAS, T.S., HARIDASAN, M., SILVA-JÚNIOR, M.C., MENDONÇA, R.C. \& RESENDE, A.V. 1994. Projeto Biogeografia do Bioma Cerrado: vegetação e solos. Cad. Geo. IBGE 12(4):75-166.

FELFILI, J. M., FAGG, C.W., SILVA, J.C.S., OLIVEIRA, E.C.L., PINTO, J.R.R., SILVA-JUNIOR, M.C. \& RAMOS, K.M.O. 2002. Plantas da APA Gama e Cabeça de Veado: espécies ecossistemas e recuperação. Universidade de Brasília, Departamento de Engenharia Florestal, Brasília.

GOODLAND, R. 1969. Análise ecológica da vegetação do cerrado. In Ecologia do cerrado (R. Goodland \& M.G. Ferri, eds.). EDUSP, São Paulo, p. 167-179.

KOVACH COMPUTING SERVICES. 2005. Multivariate statistical package. Anglesey.

McCUNE, B. \& MEFFORD, M.J. 1999. PC-ORD version 4.2, multivariate analysis of ecological data, Users guide. MiM Software Design, Glaneden Beach, Oregon.

MANTOVANI, W. \& MARTINS, F.R. 1993. Florística do Cerrado na Reserva Biológica de Moji Guaçu, SP. Acta Bot. Bras. 7(1):33-60.

MENDONÇA, R.C., FELFILI, J.M., WALTER, B.M.T., SILVA JÚNIOR, M.C., REZENDE, A.V., FILGUEIRAS, T.S. \& NOGUEIRA, P.E. 1998. Flora vascular do Cerrado. In Cerrado: Ambiente e Flora (S.M. Sano \& S.P. de Almeida, eds.). EMBRAPA-CPAC, Brasília, p. 289-556.

MUELLER-DOMBOIS, D. \& ELLENBERG, H. 1974. Aims and methods of vegetation ecology. Willey and Sons, New York.

MUNHOZ, C.B.R. \& FELFILI, J.M. 2004. Composição florística do estrato herbáceo-subarbustivo em uma área de campo sujo na Fazenda Água Limpa no Distrito Federal, Brasil. B. Herb. Ezechias Paulo Heringer 13(1):85-113.

MUNHOZ, C.B.R. \& FELFILI, J.M. 2006 a. Floristics of the herbaceous and sub-shrub layer of a moist grassland in the Cerrado Biosphere Reserve (Alto Paraíso De Goiás), Brazil. Edinb. J. Bot. 63(2\&3):343-354. 
MUNHOZ, C.B.R. \& FELFILI, J.M. 2006 b. Fitossociologia do estrato herbáceo-subarbustivo de uma área de campo sujo no Distrito Federal, Brasil. Acta Bot. Bras. 20(3):671-685.

PINDER, L. \& ROSSO, S. 1998. Classification and ordination of plant formations in the Pantanal of Brazil. Plant Ecol. 136(2):151-165.

PIRANI, J.R., GIULIETTI, A.M., MELLO-SILVA, R. \& MEGURO, M. 1994 Checklist and patterns of geographic distribution of the vegetation of Serra do Ambrósio, Minas Gerais, Brazil. Rev. Brasil. Bot. 17(2):133-147.

PRANCE, G.T., BEENTJE, H., DRANSFIELD, J. \& JOHNS, R. 2000. The tropical flora remains undercollected. Ann. Miss. Bot. Gard. 87(1):67-71.

PROENÇA, C.E.B., MUNHOZ, C.B.R., JORGE, C.L. \& NÓBREGA, M.G.G 2001. Listagem e nível de proteção das espécies de fanerógamas do Distrito Federal, Brasil. In Flora do Distrito Federal, Brasil (T.B. Cavalcanti \& A.E. Ramos, eds). EMBRAPA-CENARGEN, Brasilia, v.1, p. 87-359.

RATTER, J.A., BRIDGEWATER, S. \& RIBEIRO, J.F. 2003. Analysis of the floristic composition of the Brazilian cerrado vegetation III: Comparison of the wood vegetation of 376 areas. Edinb. J. Bot. 60:57-109.

REATTO, A., CORREIA, J.R. \& SPERA, S.T. 1998. Solos do bioma cerrado: aspectos pedológicos. In Cerrado: Ambiente e Flora (S.M. Sano \& S.P. de Almeida, eds.). EMBRAPA-CPAC, Brasília, p. 47-88.
RIBEIRO, J.F. \& WALTER, B.M.T. 1998. Fitofisionomias do Bioma Cerrado. In Cerrado: Ambiente e Flora (S.M. Sano \& S.P. de Almeida, eds.) EMBRAPA-CPAC, Brasília, p. 98-166.

SARMIENTO, G. 1992. Adaptive strategies of perennial grasses in South American savannas. J. Veg. Sci. 3(3):325-336.

SAMPAIO, A.B., WALTER, B.M.T. \& FELFILI, J.M. 2000. Diversidade e distribuição de espécies arbóreas em duas matas de galeria na micro-bacia do Riacho Fundo, Distrito Federal. Acta Bot. Bras. 14(2):197-214.

SILVA, M.A. \& NOGUEIRA, P.E. 1999. Avaliação fitossociológica do estrato arbustivo-herbáceo em cerrado stricto sensu após incêndio acidental, no Distrito Federal, Brasil. Bol. Herb. Ezechias Paulo Heringer 4:65-78.

SILVA JUNIOR, M.C. da. 2001. Comparação entre matas de galeria no Distrito Federal e a efetividade do código florestal na proteção de sua diversidade arbórea. Acta Bot. Bras. 15(1):139-146.

TANNUS, J.L.S. \& ASSIS, M.A. 2004. Composição de espécies vasculares de campo sujo e campo úmido em área de cerrado, Itirapina - SP, Brasil. Rev. Brasil. Bot. 27(3):489-506.

THE PLANT NAMES PROJECT 2000. International Plant Names Index. Acesso em http://www.ipni.org, acesso em: março de 2007.

W³ TRÓPICOS <http://www.mobot.org> , acesso em: junho de 2007. 
